\title{
Expressions of Fas/DcR3 and RGD-FasL mediated apoptosis in pituitary adenomas
}

\author{
Lukui Chen, Xinhua Tian, W enzhu Li ${ }^{1}$, Abhishek Agarwal ${ }^{2}$, Guohong Zhuang ${ }^{1}$
}

Department of Neurosurgery, Zhongshan Hospital, Xiamen University, Xiamen, Fujian - 361 004, ${ }^{1}$ Cancer Research Center, Medical College, Xiamen University, Xiamen, Fujian - 361 005, China, ${ }^{2}$ Department of Neurosurgery, Fujita Health University, Toyoake, Japan

\author{
Address for correspondence: \\ Dr. Lukui Chen, \\ Department of Neurosurgery, \\ Zhongshan Hospital, Xiamen \\ University, 201-209, South Hubin \\ Street, Xiamen, 361 004, Fujian, \\ China. \\ E-mail: neuro_clk@ hotmail.com
}

DOI: $10.4103 / 0028-3886.48808$

\begin{abstract}
Abstrad
Purpose: To detect the expressions of Fas/DCR3 and to investigate the cytotoxic effects of RGD-FasL on pituitary adenoma cells. Materials and Methods: Fas/DcR3 mRNAs were detected by Reverse transcription polymerase chain reaction (RT-PCR) and their surface expressions were measured by flow cytometry. Cytotoxicities exerted by FasL and newly-constructed RGD - FasL on tumor cells were measured with 3-(4,5-D imethylthiazol2-yl)-2,5-diphenyltetrazolium bromide (MTT) assay. The apoptotic cells were examined by electron microscopy and the induced apoptosis was determined by agarose gel electrophoresis. The cell cycle was assessed by flow cytometry with AN N EXIN V FITC/ PI. The expressions of caspases, BCl-2, RANKL and JNK2 were detected by Western blotting. Results: Fas/DcR3 was expressed in GH3/MM Q/AtT 20 cells. The cytotoxic effects of RGD-FasL on tumor cells were seen in a dose-dependent manner. These cells showed the same sensitivity to RGD -FasL as to FasL. RGD -FasL induced apoptosis and G1/G0 arrest. The expressions of caspase-8/9/3, RANKL, JNK2 were increased while that of $\mathrm{BCl}-2$ was decreased with treatment of RGD -FasL. Conclusions: Fas can be a novel target for the treatment of pituitary adenomas. RGD-FasL induces apoptosis of pituitary adenoma cells through caspase activation.
\end{abstract}

Key words: Apoptosis, DCR3, fas, fasl, pituitary adenoma, RG D-FasL

\section{Introduction}

Novel approaches are needed to eliminate pituitary adenomas, since the invasiveness of bone; dura or cavernous sinus may adversely affect the outcome of tumors and compromise the disease-free survival of patients. Jaita et al., detected that Fas and FasL are expressed in several anterior pituitary cell types from female rats, mainly in lactotropes and somatotropes. In addition, they reported that Fas activation induces apoptosis of lactotropes and somatotropes, ${ }^{[1]}$ suggesting that FasL is involved in the maintenance of tissue homeostasis in the anterior pituitary gland and could have potential benefit for the treatment of pituitary adenomas.

\section{Materials and M ethods}

Pituitary adenoma cell lines GH3, MMQ, AtT20 were cultured and underwent RT-PCR analysis for expressions of Fas and DcR3 mRNA, followed by flow cytometry analysis for expressions of Fas and DcR3 protein. The FasL gene was constructed by overlapping PCR, then was cloned into pGEX-5X-1, which contained a GST tag. RGD was fused onto the N-terminal of FasL. The two proteins, FasL and RGD-FasL, were purified through a GST resin column (Novagen, USA). The effects of FasL/RGD-FasL on cell proliferation were measured using an MTT-based assay, in the presence of various concentrations (1.5625, $3.125,6.25,12.5,25,50 \mu \mathrm{g} / \mathrm{mL}$ ) of FasL/RGD-FasL for $12 \mathrm{~h}$. The apoptotic cells were examined by electron 
microscopy. For DNA fragmentation analysis, tumor cells were treated with RGD-FasL (3.125, 6.25, 12.5, 25, 50 $\mu \mathrm{g} /$ $\mathrm{mL}$ ) for $4 \mathrm{~h}$. Then, cell cycle distribution and apoptosis were determined by flow cytometry with ANNEXIN V FITC/PI. Western blot analysis was performed for protein expressions of caspase-8/9/3, RANKL, JNK2 and Bcl-2 (Sigma, USA). The mechanism of apoptosis was confirmed with caspase inhibition assay.

\section{Results}

There was no significant difference in the mRNA expression level of Fas between the three cell lines examined. The mRNA expression of DcR3 in At T20 cells was lower than that in GH3/MMQ cells. Approximately $13.7 \%$ of GH3 cells, $25.5 \%$ of MMQ cells, $22.2 \%$ of AtT20 cells expressed Fas protein, while about 23.9\% of GH3 cells, 24.1\% of MMQ cells, $4.6 \%$ of AtT20 cells expressed DcR3 protein. The cytotoxic effects of RGD-FasL on tumor cells were seen in a dose-dependent manner. These cells showed the same sensitivity to RGD-FasL as to FasL. Electron microscopy showed that GH3 cells treated with RGD-FasL $(50 \mu \mathrm{g} / \mathrm{ml})$ for $4 \mathrm{~h}$ caused the shrinkage and condensation of nucleus and cytoplasm, the margination of chromatin along with nuclear membrane, and the formation of apoptotic bodies [Figure 1]. After treatment of GH3 cells with RGD-FasL at various concentrations for $4 \mathrm{~h}$, a typical ladder pattern of inter-nucleosomal DNA fragmentation was detected using an agarose gel electrophoresis [Figure 2]. The cell death mode induced by RGD-FasL was assessed by Flow cytometry with ANNEXIN V FITC kit. The apoptotic cells increased significantly in both GH3 cell line and AtT20 cell line [Figure 3]. And the expressions of caspase-8/9/3, RANKL, JNK2 were increased while that of Bcl-2 was decreased [Figure 4].
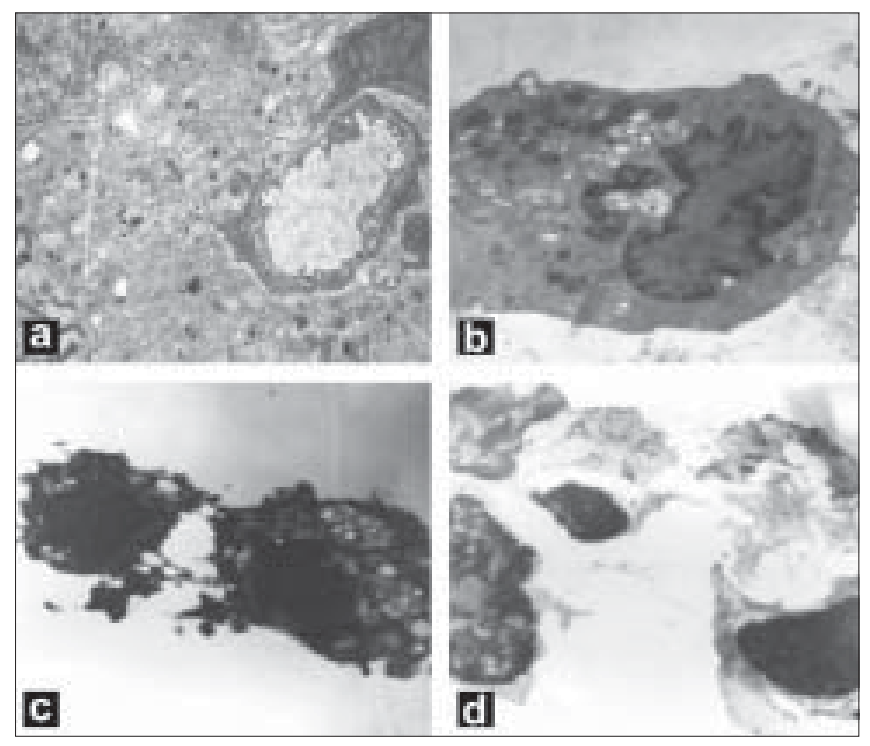

Figure 1: Electron microscopy (a) untreated GH3 cell; (b-d) $\mathrm{GH} 3$ cells treated with RGD-FasL $(50 \mu \mathrm{g} / \mathrm{ml})$ for $4 \mathrm{~h}$
The caspase inhibitor, Z-VAD-FMK, could inhibit the apoptosis induced by RGD-FasL.

\section{Discussion}

The $\alpha_{v} \beta_{3}$ integrin is one of the most specific markers for targeted delivery of cytotoxic agents to the tumor vasculature. ${ }^{[2]}$ In our study, the high affinity $\alpha_{v} \beta_{3}$ selective ligand, Arg-Gly-Asp (RGD) peptide was used for newly-developed RGD-FasL (produced by our team), which allows active targeting and delivery of FasL to sites of tumor angiogenesis devoid of systemic toxicity. There have been a series of inspiring tests showing that RGD-FasL exerts cytotoxic activity on U138 and Hela cell lines more than FasL (data not shown).

It was demonstrated that the somatolactotrophic tumor cell line GH3 and the corticotrophic tumor cell line AtT20 express Fas. ${ }^{[3,4]}$ In GH3 and AtT20 cells, overexpression of Fas strongly enhanced cell death by apoptosis. Considering that apoptosis plays a central role in regulation of pituitary tissue homeostasis, the imbalance between cell death and proliferation in favor of cell survival could result in pituitary adenoma formation. Whether the existence and difference of expressions of Fas/DcR3 on tumor cells could be the cause of selective apoptosis induced by FasL remains unclear and needs to be determined further.

Our results demonstrate that functionally, RGD-FasL has the same effective cytotoxicity as the FasL does on pituitary adenoma cells; RGD-FasL induces apoptosis through caspase activation. We conclude that RGD-FasL could probably be considered as a novel therapeutical candidate for the treatment of pituitary adenomas. Further studies are currently underway on animal models to determine the targeted cytotoxicity of RGDFasL towards tumor-associated vasculature and tumor

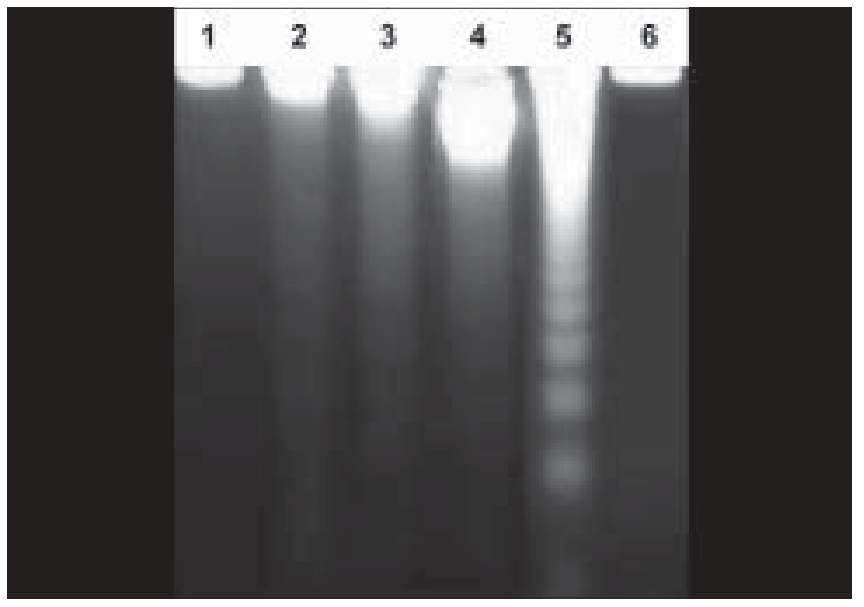

Figure 2: GH3 cells were treated with various concentrations (Lane 1-5 represent 3.125, 6.25, 12.5, 25, $50 \mu \mathrm{g} / \mathrm{ml}$ ) of RGD-FasL. After $4 \mathrm{~h}$, fragmented DNA was collected and separated by agarose gel electrophoresis and visualized by ethidium bromide staining 


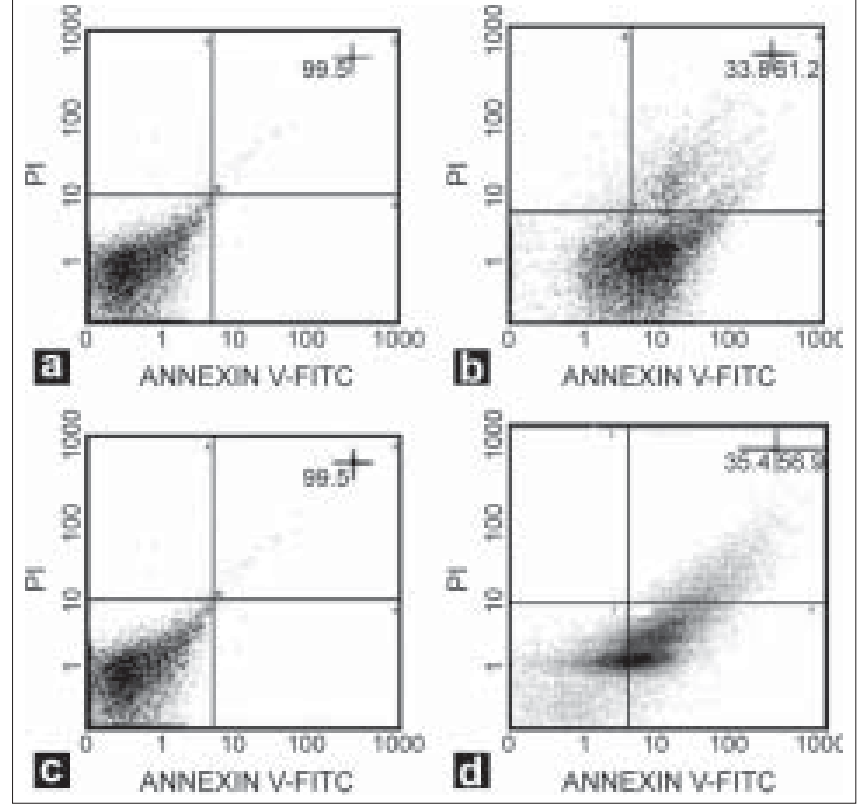

Figure 3: Flow cytometric analysis of cell death mode induced by RGDFasL with ANNEXIN V FITC/PI. (a) untreated GH3 cells; (b) treated GH3 cells; (c) untreated AtT20 cells; (d) treated AtT20 cells

cells. Part of our further studies suggests that pituitary tumor stem cells might be self-existent and RGD-FasL may probably target pituitary tumor stem cells and suppress the tumor growth through $\alpha_{\mathrm{v}} \beta_{3}$.

\section{Acknowledgment}

This work was supported by grants from Zhongshan Hospital of Xiamen University, and from Health Bureau of Xiamen, China.

\section{Abbreviations}

FasL: Fas ligand

DcR: decoy receptor

RGD: tripeptide sequence Arg-Gly-Asp

RGD-FasL: fusion protein of RGD and Fas ligand

GH: Growth hormone

RT-PCR: reverse transcription polymerase chain reaction

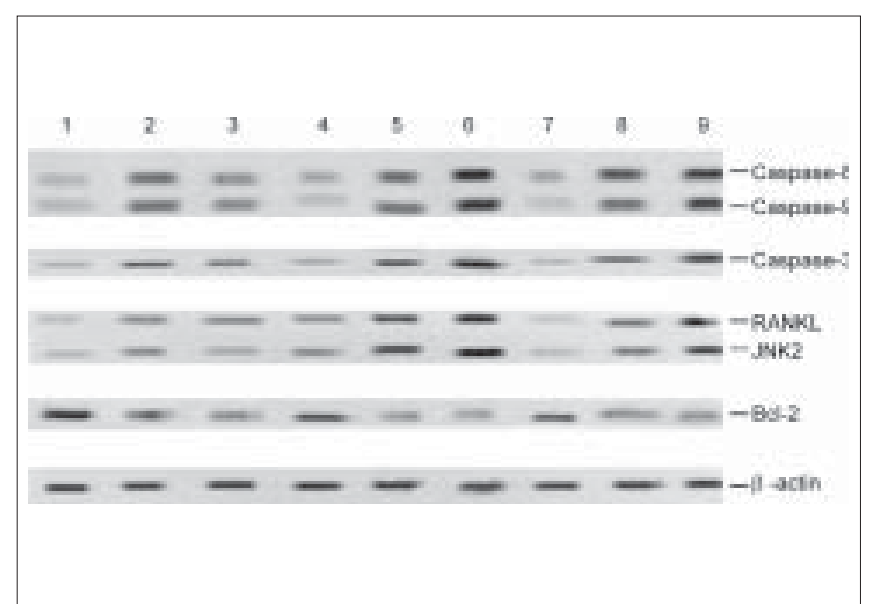

Figure 4: Detection of levels of caspase-8/9/3, Bcl-2, RANKL, JNK2 protein expression in 3 cells lines by Western blot analysis. Lane 1: GH3; Lane 2: GH3+FasL; Lane 3: GH3+RGD-FasL; Lane 4: MMQ; Lane 5: MMQ +FasL; Lane 6: MMQ +RGD-FasL; Lane 7: AtT20; Lane 8: AtT20 +FasL; Lane 9: AtT20 +RGD-FasL

MTT: 3-(4,5-Dimethylthiazol-2-yl)-2,5-

diphenyltetrazolium bromide

JNK: c-Jun N-terminal kinase

RANKL: Receptor activator of nuclear factor kappa B ligand

Bcl-2: B-cell lymphoma leukemia 2

\section{References}

1. Jaita G, Candolfi M, Zaldivar V, Zarate S, Ferrari L, Pisera D, et al. Estrogens up-regulate the Fas/FasL apoptotic pathway in lactotropes. Endocrinology 2005;146:4737-44.

2. Arap W, Pasqualini R, Ruoslahti E. Cancer treatment by targeted drug delivery to tumor vasculature in a mouse model. Science 1998;279: 377-80.

3. Kobayashi H, Fukata J, Murakami N, Usui T, Ebisui O, Muro S, et al. Tumor necrosis factor receptors in the pituitary cells. Brain Res 1997;758:45-50.

4. Huang P, Tofighi R, Emgard M, Ceccatelli S. Cell death induced by 2,3,7,8-tetrachlorodibenzo-p-dioxin (2,3,7,8-TCDD) in AtT-20 pituitary cells. Toxicology 2005;207:391-9.

Accepted on 01-02-2009

Source of Support: Nil, Conflict of Interest: None declared. 